DIAS-STP-03-09

\title{
Fuzzy Complex Quadrics and Spheres
}

\author{
Brian P. Dolan, ${ }^{a), b) *}$ Denjoe O'Connor ${ }^{b) \dagger}$ and P. Prešnajder ${ }^{c) \ddagger}$
}

a) Dept. of Mathematical Physics, NUI, Maynooth, Ireland

b) School of Theoretical Physics, Dublin Institute for Advanced Studies, 10 Burlington Rd., Dublin 8, Ireland

c) Dept. of Theoretical Physics, Comenius University, Mlynská dolina, SK-84248 Bratislava, Slovakia

January 17,2004

\begin{abstract}
A matrix algebra is constructed which consists of the necessary degrees of freedom for a finite approximation to the algebra of functions on the family of orthogonal Grassmannians of real dimension $2 N$, known as complex quadrics. These matrix algebras contain the relevant degrees of freedom for describing truncations of harmonic expansions of functions on $N$-spheres. An Inönü-Wigner contraction of the quadric gives the co-tangent bundle to the commutative sphere in the continuum limit. It is shown how the degrees of freedom for the sphere can be projected out of a finite dimensional functional integral, using second-order Casimirs, giving a well-defined procedure for construction functional integrals over fuzzy spheres of any dimension.
\end{abstract}

*bdolan@thphys . may . ie

$\dagger$ denjoe@stp.dias.ie

${ }_{\ddagger}^{\ddagger}$ presnajder@fmph. uniba.sk 


\section{Introduction}

Non-commutative geometry has slowly been increasing in importance in physics over the last 20 years and has recently received a strong impetus through work in string theory. An important concept in the non-commutative programme is that of a "fuzzy" space - this is perhaps more correctly described as a finite, non-commuting, matrix approximation to the algebra of functions on a continuous manifold which can reproduce the commutative algebra in the limit of the matrices becoming infinite in size. It has been suggested that fuzzy spaces could provide a regularisation technique for numerical calculations in quantum field theory which would be an alternative to lattice gauge theory, [1]-[4]. The prototypical example of a fuzzy space is the fuzzy two-sphere, [5], but there are many more examples, and indeed much of the work on generalised coherent states in quantum mechanics [6], when restricted to compact groups, can be related to fuzzy spaces. The geometry of fuzzy $C P^{N}$ and fuzzy unitary Grassmannians has been examined in the literature in some detail [7]-[10].

Clearly, from the point of view of a regularisation technique in field theory as well as for the theory of $D$-branes in string theory, it would be desirable to have an explicit construction of the fuzzy sphere $S_{F}^{N}$ in dimensions other than $N=2$. Unfortunately, despite the elegant simplicity of the fuzzy two-sphere $S_{F}^{2}$, higher dimensional spheres are not so amenable to a fuzzy description. To our knowledge there is no closed finite matrix approximation to the algebra of functions on a sphere for dimensions greater than two, though the problem was tackled in [11] and [12] (non-commutative spheres in the continuum were analysed in [13]). It appears that, while one can represent truncated harmonic expansions of functions on spheres by square matrices, the product of two such matrices takes one out of the required space and a projection back into the space of functions is necessary after every multiplication - this renders the product non-associative. In other words a star product cannot be defined on the fuzzy sphere $S_{F}^{N}$ for $N>2$ (this is related to the fact that, for $N \neq 2, S^{N}$ cannot be obtained as the co-adjoint orbit of a compact group). Nevertheless the construction in [11] does associate a square matrix with the truncation of a harmonic expansion on $S^{N}$ and so does, in a sense, constitute a fuzzy sphere, even though there is no associative product.

For $S_{F}^{4}$ alternative descriptions are possible, [14] [15]. The construction in [15] is in terms on $C P_{F}^{3}$ and uses the fact that the continuum $C P^{3}$ is an $S^{2}$ bundle over $S^{4}$ [15]. This approach has the advantage that it is designed to be used in a functional integral and the technique was extended to $S_{F}^{3}$ and $S_{F}^{1}$ in [16]. The method of [11] would be particularly cumbersome to implement in functional integrals over $S_{F}^{3}$ and $S_{F}^{1}$, or indeed any odd sphere. 
In this paper we generalise the construction in [16] to fuzzy spheres of any dimension. The analysis relies on properties of the orthogonal Grassmannian, $S O(N+2) /[S O(N) \times S O(2)]$, which is a co-adjoint orbit of dimension $2 N$. This orthogonal Grassmannian can also be obtained as the complex quadratic $z^{a} z^{a}=0$ in $C P^{N+1}$, where $z^{a}$ are natural complex co-ordinates, [17]. The notation in Kobayashi and Nomizu is $Q^{N} \cong S O(N+2) /[S O(N) \times S O(2)]$ and we shall use this as a shorthand. Inönü-Wigner contraction of $S O(N+2)$ to the Euclidean group of $\mathbf{R}^{N+1}$ relates $Q^{N}$ to the co-tangent bundle $T^{*} S^{N}$. In a sense, elaborated on in section $2, Q^{N}$ can be thought of as the compactified co-tangent bundle for a non-commutative sphere. There is a finite dimensional, non-commutative, matrix algebra approximation to the algebra of functions $Q^{N}$ which contains harmonic expansions on $S^{N}$ (described in section 3). While it is still the case that the resulting matrix algebra takes one out of the space of harmonic expansions on $S^{N}$ upon multiplication (so one does not have a closed matrix approximation to the algebra of functions on $S^{N}$ for $N \neq 2$ ) it is nevertheless very easy to suppress unwanted modes in a functional integral over a fuzzy complex quadric, $Q^{N}$, in a manner which lends itself naturally to numerical computation for field theory on $S_{F}^{N}$, as described in section 4 .

\section{The deformed co-tangent bundle}

In this section it is shown how the co-tangent bundle $T^{*} S^{N}$ can be deformed to a version related to a non-commutative sphere and compactified to complex quadric, $Q^{N}$.

The construction starts with Cartesian co-ordinates $X^{a}$ in $\mathbf{R}^{N+1}$, where $a=1, \ldots, N+1$. Consider the sphere $S^{N} \cong S O(N+1) / S O(N)$ of radius $R$ defined by $X^{a} X^{a}=R^{2}$. The isometry group is $S O(N+1)$ with algebra

$$
\left[L_{a b}, L_{c d}\right]=i\left(\delta_{b c} L_{a d}+\delta_{a d} L_{b c}-\delta_{a c} L_{b d}-\delta_{b d} L_{a c}\right),
$$

where $L_{a b}=-L_{b a}$ (there is no distinction between upper and lower Euclidean indices in $\mathbf{R}^{N+1}, X^{a}=X_{a}$ ). This can be extended to a representation of the Euclidean group, $E_{N+1}$ acting on $\mathbf{R}^{N+1}$,

$$
\begin{aligned}
{\left[L_{a b}, L_{c d}\right] } & =i\left(\delta_{b c} L_{a d}+\delta_{a d} L_{b c}-\delta_{a c} L_{b d}-\delta_{b d} L_{a c}\right) \\
{\left[L_{a b}, X_{c}\right] } & =i\left(\delta_{b c} X_{a}-\delta_{a c} X_{b}\right) \\
{\left[X_{a}, X_{b}\right] } & =0 .
\end{aligned}
$$

An explicit realisation of this algebra in the continuum is

$$
L_{a b}=i\left(X_{a} \frac{\partial}{\partial X_{b}}-X_{b} \frac{\partial}{\partial X_{a}}\right)
$$


acting on functions and $X_{a}$ being commutative multiplication by the coordinates.

The algebra will now be deformed, essentially using the inverse of InönüWigner contraction. Let $X_{a}:=\mu L_{a, N+2}$, with $\mu$ real, and replace the commutator (4) above with

$$
\left[X_{a}, X_{b}\right]=-i \mu^{2} L_{a b}
$$

leaving the other commutators unchanged. The Euclidean group $E_{N+1}$ is thus deformed to $S O(N+2)$

$$
\left[L_{A B}, L_{C D}\right]=i\left(\delta_{B C} L_{A D}+\delta_{A D} L_{B C}-\delta_{A C} L_{B D}-\delta_{B D} L_{A C}\right),
$$

where $A, B, C, D=1, \ldots, N+2$ and $L_{a, N+2}=-L_{N+2, a}$. In terms of the quadratic Casimirs ${ }^{1} C_{2}^{N+1}=(1 / 2) L_{a b} L_{a b}$ and $C_{2}^{N+2}=(1 / 2) L_{A B} L_{A B}$ we see that

$$
X_{a} X_{a}=\mu^{2}\left(C_{2}^{N+2}-C_{2}^{N+1}\right) .
$$

While it may be tempting to think of $X^{a}$ in (8) as co-ordinates on a fuzzy sphere we must be careful: a general irreducible representation of $S O(N+2)$ will decompose into a sum of different irreducible representations of $S O(N+1)$, with different values of $C_{2}^{N+1}$, so the right-hand side of (8) will not be central. However $X_{a} X^{a}$ is central in the fundamental spinor representation of $\operatorname{Spin}(N+2)$. To see this consider the even and odd cases separately:

- Even $N$ : choose one chirality of spinor with $2^{N / 2}$ components. Under $\operatorname{Spin}(N+2) \rightarrow \operatorname{Spin}(N+1)$ this reduces uniquely to the single spinor representation of $\operatorname{Spin}(N+1)$, which has the same dimension. In this case the right-hand side of (8) is a multiple of the identity.

- Odd $N$ : in this case the $2^{N / 2}$ dimensional spinor representation of $\operatorname{Spin}(N+2)$ decomposes into two spinor representations of opposite chirality under $S O(N+2) \rightarrow S O(N+1)$, both of dimension $2^{(N / 2)-1}$. But the second order Casimir $C_{2}^{N+1}$ has the same value on the two chiralities, it does not distinguish between them, [18]. So again the right-hand side of (8) is a multiple of the identity.

The fundamental spinor representations of $\operatorname{Spin}(k)$ have quadratic Casimir $C_{2}^{k}=k(k-1) / 4$ for both even and odd $k$, see [18] for example. For a fundamental spinor representation of $\operatorname{Spin}(N+2)$ equation (8) therefore gives

$$
X_{a} X^{a}=\frac{\mu^{2}(N+1)}{2} \mathbf{1}
$$

\footnotetext{
${ }^{1}$ Relative to the standard conventions for $S U(n)$ our normalisation here is such that $C_{2}^{S U(2)}=\frac{1}{2} C_{2}^{3}$ and $C_{2}^{S U(4)}=\frac{1}{2} C_{2}^{6}$.
} 
where $\mathbf{1}$ is the identity operator, and we might interpret $\sqrt{\frac{N+1}{2}} \mu$ as the radius of a fuzzy sphere. However, unlike $S_{F}^{2}$, the matrix algebra generated by $X_{a}$ will not close in general even when $X_{a} X^{a}$ is central. Also one cannot get higher dimensional representations of $S_{F}^{N}$ by taking tensor products. This reflects the fact that there is no closed finite matrix approximation to $S_{F}^{N}$ for $N \neq 2$.

Let us now investigate the geometry of the space generated by the adjoint action of $\operatorname{Spin}(N+2)$ on a fiducial direction $X_{N+1}$ (the "north pole" of $\left.S_{F}^{N}\right)$. Clearly $\left[L_{\alpha \beta}, X_{N+1}\right]=0$ for $\alpha, \beta=1, \ldots, N$, so $\operatorname{Spin}(N)$ leaves $X_{N+1}$ invariant. Also $L_{N+1, N+2}$ commutes with $X_{N+1}$, since they are just multiples of one another, so the $S O(2)$ generated by $L_{N+1, N+2}$ also leaves $X_{a}$ invariant. The upshot of this is that the manifold that $D(g) \in \operatorname{Spin}(N+2)$ generates with the action

$$
D^{-1}(g) X_{N+1} D(g)
$$

is the orthogonal Grassmannian $Q^{N}$, which has dimension $2 N$. For $N \leq 4$ these spaces have the following structures:

- $Q^{1} \cong S O(3) / S O(2) \cong S^{2}$;

- $Q^{2} \cong S O(4) /[S O(2) \times S O(2)] \cong S^{2} \times S^{2}\left(S^{2} \times S^{2}\right.$ was used in a consideration of fuzzy $S^{3} / \mathbf{Z}_{2}$ in [19]);

- $Q^{3} \cong S O(5) /[S O(3) \times S O(2)] \cong C P^{3} / \mathbf{Z}_{2}$ (this identification is described in [16]);

- $Q^{4} \cong S O(6) /[S O(4) \times S O(2)] \cong S U(4) /[S(U(2) \times U(2))]$ (matrix approximations to this space were described in $[10])$.

We propose to identify $Q^{N}$ with a 'compactified' co-tangent bundle for a non-commutative sphere. This reduces to the usual $T^{*} S^{N}$ under InönüWigner contraction $\mu \rightarrow 0$ : this limit performs the dual function of rendering the $X$ 's in (6) commutative while at the same time de-compactifying $S O(N+2)$ to the Euclidean group $E_{N+1}$.

\section{$3 \quad$ Fuzzy Complex Quadrics, $Q_{F}^{N}$}

Any unitary irreducible representation $T$ of a simple compact Lie group is finite dimensional and the extension to its enveloping algebra is a finite dimensional matrix algebra. This matrix algebra provides a fuzzy approximation to the algebra of functions on the co-adjoint orbit of any Lie algebra element in $T$. 
We shall refer to a finite matrix approximation to $Q^{N}$ as a fuzzy complex quadric and denote it by $Q_{F}^{N}$. Being a co-adjoint orbit $Q^{N}$ is a symplectic manifold whose algebra of functions can be approximated by closed finite dimensional matrix algebras. The harmonic expansion of a function on $Q^{N}$ requires all representations of $S O(N+2)$ which contain the trivial representation under $S O(N) \times S O(2)$. A spinor representation of $S O(N+2)$ never contains a singlet of $S O(N) \times S O(2)$ for either even or odd $N$, so we restrict to vectorial representations. The representation space of $S O(N+2)$ is then the space of rank- $n$ tensors, $T_{A_{1} \cdots A_{n}}$. Irreducible representations can be characterised by their symmetries under interchange of their indices and are traceless in any two indices. They can be represented by Young tableau with $n$ boxes which reflect their permutation symmetries.

Any tensor that is anti-symmetric in three or more of its indices must vanish when restricted to a representation of $S O(2)$ and hence cannot contribute to the harmonic expansion of functions on $Q^{N}$. Thus we can restrict our attention to tensors that are anti-symmetric in pairs of indices only. If $T_{A_{1} \cdots A_{n}}$ has $m$ pairs of anti-symmetric indices and $n-2 m$ symmetric indices then the corresponding Young tableau is

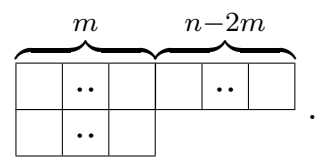

When $S O(N+2)$ is restricted to $S O(N) \times S O(2)$ the anti-symmetric pairs all contain singlets of $S O(N) \times S O(2)$ (when both indices in a pair are $S O(2)$ indices, for example). Also if $n$ is even the $n-2 m$ symmetric indices can contain singlets of $S O(N) \times S O(2)$, but not when $n$ is odd. Thus the harmonic expansion of a function on $Q^{N}$ requires all $S O(N+2)$ tensor representations of the form (11) with $n$ even. The dimension of these representation can be determined, using the relevant formulae in [18] for example. With $n=2 l$ they are, for $N \geq 3$,

$$
\begin{gathered}
d_{N}(2 l, m)=(2 l+N-1)(2 l+1-2 m)(4 l+N-2 m)(N-2+2 m) \\
\times \frac{(2 l+N-2-m) !(N-3+m) !}{N !(N-2) !(2 l-m+1) ! m !}
\end{gathered}
$$

where $m \leq l .{ }^{2}$ If the harmonic expansion of a function on $Q^{N}$ is truncated at $l_{\max }=L$ the total number of degrees of freedom is

$$
\sum_{l=0}^{L} \sum_{m=0}^{l} d_{N}(2 l, m)=\left[\frac{(2 L+N)(L+N-1) !}{L ! N !}\right]^{2}=\left[d_{N}(L, 0)\right]^{2} .
$$

\footnotetext{
${ }^{2}$ In the notation of [18] the representations (11) have highest weights $\left(r_{1}, \ldots, r_{[N / 2]+1}\right)=(n-m, m, 0, \ldots, 0)$, where $[N / 2]$ is the integer part of $N / 2$.
} 
The fact that this is a perfect square reflects the fact that the degrees of freedom in a truncated harmonic expansion can be arranged into a square matrix of size $d_{N}(L, 0) \times d_{N}(L, 0)$. This can be represented in terms of Young tableau by

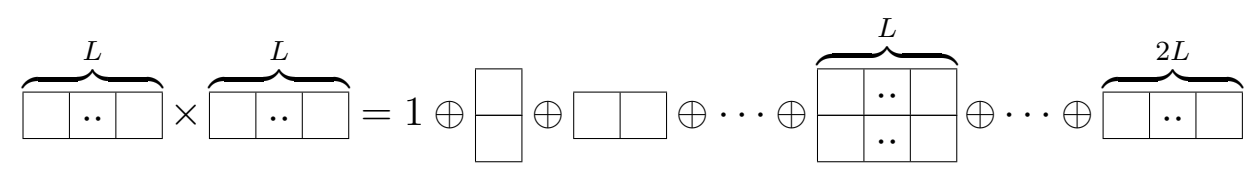

which relates the matrix structure, on the left-hand side, to the harmonic expansion, on the right-hand side. Matrix multiplication then gives a closed associative, but non-commutative, algebra which reproduces the commutative algebra of functions on $Q^{N}$ as $L \rightarrow \infty$. At the level of functions the non-commutative product at finite $L$ can be realised as a $*$-product for the fuzzy complex quadric, $Q_{F}^{N}$.

\section{Functional Integrals on Fuzzy Spheres, $S_{F}^{N}$}

There is no closed finite matrix approximation for the truncated algebra of functions on $S^{N}$ known, except for the special case $N=2$ which was first described in [5]. There does exist a matrix approximation to functions on $S^{N}$, but in general matrix multiplication does not correspond to the algebra of functions and the latter can only be recovered by projecting back onto a function on $S^{N}$ after matrix multiplication [11]. This results in a nonassociative algebra when $N \neq 2$ which, by a slight abuse of language, is nevertheless still referred to as a "fuzzy" sphere, $S_{F}^{N}$.

In the construction presented here a similar projection can be performed, since the truncated harmonic expansion of a function on $S^{N}$ is buried in $Q_{F}^{N}$. To see this note that functions on $S^{N}$ can be expanded in symmetric tensor representations of $S O(N+1)$. A truncation at level $l_{\max }$ requires using all symmetric tensors of $S O(N+1), T_{a_{1} \cdots a_{l}}$, with $0 \leq l \leq l_{\max }$. These are all contained in one symmetric representation

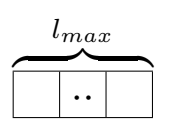

of $S O(N+2)$ under $S O(N+2) \rightarrow S O(N+1)$. Setting $l_{\max }=2 L$ we see that the last representation on the right-hand side of (14) contains all the $S O(N+1)$ representations necessary for the harmonic expansion of a function on $S^{N}$ up to angular momentum $2 L$. We can thus obtain the fuzzy sphere $S_{F}^{N}$ by projecting the irreducible representation $\overbrace{\overbrace{\mid} \cdot . \cdot}^{2 L}$ out from the matrix algebra of $Q_{F}^{N}$ in (14). 
This can be achieved in a functional integral over $Q_{F}^{N}$ in the same manner as in [16]. Let $\Phi$ be a matrix in the algebra of $Q_{F}^{N}$ for a given $L$. The $S O(N+2)$ invariant Laplacian on $Q_{F}^{N}$ is

$$
\mathcal{L}_{(N+2)}^{2} \Phi=-(1 / 2)\left[L_{A B},\left[L_{A B}, \Phi\right]\right] .
$$

Then the action for a scalar field on $Q_{F}^{N}$ can be written as

$$
S[\Phi]=\frac{1}{d_{N}(L, 0)} \operatorname{Tr}\left\{\Phi^{\dagger} \mathcal{L}_{(N+2)}^{2} \Phi+V(\Phi)\right\} .
$$

with the scalar potential $V\left(\Phi^{\dagger}\right)=V(\Phi)$ assumed bounded below. A functional integral then involves

$$
Z=\int \mathcal{D} \Phi \mathrm{e}^{-S[\Phi]}
$$

We focus on the $S_{F}^{N}$ embedded in $Q_{F}^{N}$ by penalising all the $S O(N+2)$ representations in $Z$ except the last one on the right-hand of (14). This can be achieved by modifying the kinetic term in the action. The second order Casimir of the representation (11), with $n=2 l$, is [18]

$$
C_{2}^{N+2}(2 l, m)=(2 l-m)(2 l-m+N)+m(m+N-2) .
$$

Observe that the completely symmetric tensors with $m=0$ have the largest Casimir for any given $l$,

$$
C_{2}^{N+2}(2 l, 0)=2 l(2 l+N) .
$$

Hence the operator

$$
-\left(\frac{1}{2}\right)\left[L_{A B},\left[L_{A B}, \cdot\right]-2 L(2 L+N)=C_{2}^{N+2}-2 L(2 L+N)\right.
$$

acting on $\Phi$ is negative for all modes in $\Phi$ except for the top mode, with $l=L$ and $m=0$, on which it vanishes. The $S O(N+1)$ invariant Laplacian on $S_{F}^{N}$ would be

$$
\mathcal{L}_{(N+1)}^{2} \Phi=-(1 / 2)\left[L_{a b},\left[L_{a b}, \Phi\right]\right]
$$

So the action

$S_{h}[\Phi]=\frac{1}{d_{N}(L, 0)} \operatorname{Tr}\left\{\Phi^{\dagger} \mathcal{L}_{(N+1)}^{2} \Phi+h \Phi^{\dagger}\left(-\mathcal{L}_{(N+2)}^{2}+2 L(2 L+N)\right) \Phi+V(\Phi)\right\}$,

with $h \gg 1$, will suppress all the unwanted modes in a functional integral and leave the required modes for $S_{F}^{N}$ unaffected. In the limit $h \rightarrow \infty$ all modes, except the ones relevant to $S_{F}^{N}$, will be suppressed and correlation functions calculated with

$$
Z=\int \mathcal{D} \Phi \mathrm{e}^{-S_{\infty}[\Phi]}
$$

will be those of the fuzzy sphere, truncated at level $2 L$. 


\section{Conclusions}

By constructing fuzzy approximations to complex quadrics, $Q_{F}^{N}$, a prescription for defining functional integrals over finite approximations to spheres has been presented. Although finite matrix approximations to the algebra of functions on $N$-dimensional spheres are not known for $N \neq 2$, one can construct matrix approximations to the functions, but matrix multiplication then takes one out of the space of functions on the sphere.

The construction presented here relies in the fact that the complex quadrics (which are orthogonal Grassmannians $Q^{N} \cong S O(N+2) /[S O(N) \times S O(2)]$ ) are co-adjoint orbits, and hence do have finite matrix approximations to their algebra of functions, $Q_{F}^{N}$. These spaces are related to the co-tangent bundles $T^{*} S^{N}$ — they are in a sense compactified versions of the co-tangent bundles, compactified at the expense of introducing a non-commutativity on the sphere. The algebra of functions on $Q_{F}^{N}$ contains the relevant degrees of freedom for a truncated harmonic expansion of a function on $S^{N}$. The functional integral for a field theory defined on $Q^{N}$ can be regularised in a manner that preserves the isometries and avoids Fermion doubling [20] by defining it over the fuzzy space $Q_{F}^{N}$. By modifying the kinetic term and using the action (23) the degrees of freedom that are not relevant to the underlying sphere can be prevented from contributing to the functional integral and the result is a well defined, finite approximation for the functional integral of a quantum field theory on $S^{N}$. The correct algebra is ensured by restricting $\Phi$ to be matrices of size $d_{N}(L, 0)$ given in (13) and the continuum is recovered in the limit $L \rightarrow \infty$. This construction is well suited to numerical evaluation.

\section{References}

[1] H. Grosse, C. Klimčík and P. Prešnajder, Int. J. Theor. Phys. 35, (1996) 231, [hep-th/9505175]; H.Grosse and A.Strohmaier, Lett. Math. Phys. 48, (1999) 163, [hep-th/9902138]

[2] H. Grosse, C. Klimčík and P. Prešnajder, Comm. Math. Phys. 178, (1996) 507; H. Grosse and P. Prešnajder, Lett. Math. Phys. 46, (1998) 61

[3] P. Prešnajder, J. Math. Phys. 41 (2000) 2789, [hep-th/9912050]

[4] A.P. Balachandran and S. Vaidya, Int. J. Mod. Phys. A16, (2001) 17, [hep-th/9910129]; A. P. Balachandran, T. R. Govindarajan and B. Ydri, Mod. Phys. Lett A15 (2000) 1279, [hep-th/9911087]; 
A. P. Balachandran, T. R. Govindarajan and B. Ydri, Mod. Phys. Lett. A15, (2000) 1279, [hep-th/0006216]; A. P. Balachandran, X. Martin and D. O'Connor, and Int. J. Mod. Phys. A 16 (2001) 2577, [hep-th/0007030]; S. Baez, A. P. Balachandran, S. Vaidya and B. Ydri, Comm. Math. Phys. 208, (2000) 787, [hep-th/9811169]; G. Alexanian, A.P. Balachandran, G.Immirzi and B.Ydri, J. of Geom. and Phys. 42 (2002) 28, [hep-th/0103023]

[5] J. Madore, Class. Quant. Grav. 9 (1992) 69

[6] A. M. Perelomov, "Generalized Coherent States and their Applications", Springer (1986);

[7] M. Bordemann, M. Brischle, C Emmrich and S. Waldmann, J. Math. Phys. 37 (1996) 6311, [q-alg/9512019]; Lett. Math. Phys. 36 (1996) 357, [q-alg/950300])

[8] A.P Balachandran, B.P, Dolan, J. Lee, X. Martin and D. O'Connor, J. Geom. and Phys. 43 (2002) 184 [hep-th/0107099]

[9] J. Schirmer, "A Star Product for Complex Grassmann Manifolds", [q-alg/9709021]

[10] B. P. Dolan and O. Jahn, "Fuzzy Complex Grassmannian Spaces and their Star Products", J. Phys. A18 (2003) 1935, [hep-th/0111020]

[11] S. Ramgoolam, Nucl. Phys. B610 (2001) 461, [hep-th/0105006]

[12] Yusuke Kimura, Nucl. Phys. B664 (2003) 512, [hep-th/0301055]

[13] A. Connes and G. Landi, Commun. Math. Phys. 221 (2001) 141 [math.Q/A0011194]; A. Connes and M. Dubois-Violette, "Noncommutative finite dimensional manifolds I: Spherical manifolds and related examples", [math.QA/0107070]

[14] H. Grosse, C. Klimčík and P. Prešnajder, Comm. Math. Phys. 180 (1996) 429, [hep-th/9602115)

[15] Denjoe O'Connor and Julieta Medina, "Scalar Field Theory on Fuzzy $S^{4 \prime,},[$ hep-th/0212170)]

[16] B. P. Dolan and Denjoe O'Connor, JHEP 10 (2003) 60, [hep-th/0306231] 
[17] Shoshichi Kobayashi and Katsumi Nomizu, "Foundations of Differential Geometry", Vol.II, Wiley (1996), p.278 (example 10.6)

[18] W. Fulton and J. Harris , "Representation Theory: A first course", Springer (Graduate Texts in Mathematics, No.129), (1991)

[19] V.P. Nair and S. Randjbar-Daemi, "Quantum Hall effect on $S^{3}$, edge states and fuzzy $S^{3} / \mathbf{Z}_{2}$ ", [hep-th/0309212]

[20] A. P. Balachandran, T. R. Govindarajan and B. Ydri, Mod. Phys. Lett. A15 (2000) 1279, [hep-th/9911087]; A. P. Balachandran, T. R. Govindarajan and B. Ydri "Fermion doubling problem and noncommutative geometry II", [hep-th/0006216]; A. P. Balachandran and G. Immirzi, "The Fuzzy Ginsparg-Wilson Algebra: A Solution of the Fermion Doubling Problem", [hep-th/0301242] 